\title{
MicroRNA composition of plasma extracellular vesicles: a harbinger of late cardiotoxicity in long- term survivors of acute lymphoblastic leukemia treated with doxorubicin
}

Justyna Totoń-Żurańska ( $\nabla$ justyna.toton-zuranska@uj.edu.pl )

Jagiellonian University Medical College

Joanna Sulicka-Grodzicka

Jagiellonian University Medical College

Michal Seweryn

Jagiellonian University Medical College

Ewelina Pitera

Jagiellonian University Medical College

Przemyslaw Kapusta

Jagiellonian University Medical College

Paweł Konieczny

Jagiellonian University Medical College

Leszek Drabik

Jagiellonian University Medical College

Maria Kołton-Wróż

Jagiellonian University Medical College

Bernadeta Chyrchel

Jagiellonian University Medical College

\section{Ewelina Nowak}

Jagiellonian University Medical College

Andrzej Surdacki

Jagiellonian University Medical College

Tomasz Grodzicki

Jagiellonian University Medical College

Pawel Wołkow

Jagiellonian University Medical College 
Keywords: micro-RNAs (miRNAs), Extracellular Vesicles (EVs), Cardiotoxicity, Doxorubicin, childhood acute lymphoblastic leukemia (ALL)

Posted Date: September 15th, 2021

DOI: https://doi.org/10.21203/rs.3.rs-418155/v2

License: (c) (i) This work is licensed under a Creative Commons Attribution 4.0 International License. Read Full License 


\title{
MicroRNA composition of plasma extracellular vesicles: a harbinger of late cardiotoxicity
} in long-term survivors of acute lymphoblastic leukemia treated with doxorubicin. Justyna Totoń-Żurańska ${ }^{1, \#}$, Joanna Sulicka-Grodzicka ${ }^{2}$, Michał T. Seweryn ${ }^{1,3}$, Ewelina Pitera ${ }^{1}$, Przemysław Kapusta ${ }^{1}$, Leszek Drabik ${ }^{4,5}$, Maria Kołton-Wróż ${ }^{1}$, Bernadeta Chyrchel $^{6}$, Ewelina Nowak $^{7}$, Andrzej Surdacki ${ }^{6}$, Tomasz Grodzicki ${ }^{7}$, Paweł P. Wołkow ${ }^{1,5 \#}$

${ }^{1}$ Jagiellonian University Medical College, Center for Medical Genomics OMICRON, Krakow, Poland

2 Jagiellonian University Medical College, Department of Rheumatology, Krakow, Poland

${ }^{3}$ Department of Cancer Biology and Genetics, Center for Pharmacogenomics, College of Medicine, The Ohio State University, Columbus, OH, U.S.A.

${ }^{4}$ Jagiellonian University, Medical College and John Paul II Hospital, Krakow, Poland

${ }^{5}$ Jagiellonian University Medical College, Department of Pharmacology, Krakow, Poland

${ }^{6}$ Jagiellonian University Medical College, Second Department of Cardiology, Krakow, Poland

${ }^{7}$ Jagiellonian University Medical College, Department of Internal Medicine and Gerontology, Krakow, Poland

${ }^{*}$ Corresponding author

\begin{abstract}
The use of doxorubicin is associated with an increased risk of acute and long-term cardiomyopathy. Despite the constantly growing number of cancer survivors, little is known about the transcriptional mechanisms which progress in the time leading to a severe cardiac outcome. It is also unclear whether long-term transcriptomic alterations related to doxorubicin use are similar to transcriptomic patterns present in patients suffering from other cardiomyopathies. Here we have sequenced miRNA from total plasma and extracellular vesicles (EVs) from 66 childhood acute lymphoblastic leukemia survivors treated with doxorubicin and 61 healthy controls (254 samples in total). We analyzed processes regulated by differentially expressed miRNA and cross-validated our results with the data of patients with clinically manifested cardiomyopathies. Our analyses indicate that the circulating compartment used for inspection should be selected with particular attention. miRNAs contained within EVs may be particularly informative in terms of cardiomyopathy development and may regulate pathways related to neurotrophin, transforming growth factor beta or epidermal growth factor receptors (ErbB). Among miRNAs differentially expressed in vesicles, which additionally are also most variable
\end{abstract}


between groups, we identified those correlated with echocardiographic parameters, including miR-144-3p and miR-423-3p and respectively for plasma miRNAs: let-7g-5p, miR-16-2-3p. Among these, vesicular miR-144-3p correlates with the highest number of echocardiographic parameters and is also differentially expressed in the circulation of patients with dilated cardiomyopathy. We also show that in ALL survivors' distribution of particular miRNAs, e.g., miR-184 between plasma and EVs is different than in healthy controls. This suggests persistent alterations within the cellular sorting and exporting system.

Keywords: micro-RNAs (miRNAs), Extracellular Vesicles (EVs), Cardiotoxicity, Doxorubicin, childhood acute lymphoblastic leukemia (ALL), 


\section{Introduction}

Anthracyclines, including doxorubicin, have contributed to improved survival in childhood acute lymphoblastic leukemia (ALL) from less than $10 \%$ to $90 \%$ and are still the most widely used antineoplastic drugs worldwide ${ }^{1,2}$. However, because of the lack of specificity for cancer cells, anthracyclines can also damage healthy, non-cancer cells, causing severe complications including cardiotoxicity during chemotherapy, as well as many years after treatment cessation. Among multiple health problems, heart disease is the most common non-cancer related cause of death among cancer survivors. Lipschultz et al. proved that more than $50 \%$ of doxorubicin-treated ALL survivors exhibit abnormalities of left ventricular afterload or heart muscle contractility ${ }^{3}$ several years after treatment cessation. More recent study by Jordan et al reveals that myocardial atrophy and left ventricular mass reductions are the main contributors to heart problems developing due to anthracycline use in cancer patients ${ }^{4}$. The central dogma of anthracyclines evoked cardiomyopathy, based on acute doxorubicin action, points to oxidative stress caused by excessive amounts of reactive oxygen species (ROS) produced due to severe functional disruption of mitochondria ${ }^{5,6}$. Doxorubicin action involves also massive DNA damage including 8-oxoguanine formation, DNA intercalation, and topoisomerase 2 poisoning, with downstream doublestrand breaks (DSBs) formation ${ }^{7-9}$. As DNA lesions are repaired only partially ${ }^{8}$, these should have consequences at the transcriptomic level and may be considered as the cause of long-term treatment side effects manifested at distant time points. However, knowledge on long-term transcriptomic processes leading to health complications due to anthracycline use is very limited, despite constantly increasing number of cancer survivors ${ }^{10}$. Furthermore, the analysis of gene expression in cardiac tissues is not feasible in human subjects, thus studies on molecular aspects of doxorubicin action are mostly limited to cultured cardiomyocytes in a short time scale or to animals.

In recent years the question on usability of blood circulating factors in heart disease progression monitoring has been raised ${ }^{11}$. It has been proven that circulating miRNA may be predictors of sudden cardiac/arrhythmic death in patients with coronary artery disease ${ }^{12}$. Akat et al. show that heart- and muscle-specific circulating miRNAs increased up to 140fold in advanced heart failure, whereas in stable heart disease fold changes were lower, however miRNAs might still serve as indicators of heart muscle injury ${ }^{13}$. 
MiRNAs may circulate in blood in a form of protein-bound complexes but may also be encapsulated in extracellular vesicles (EVs) and each of these compartments may contain a different set of miRNAs or the same miRNAs yet with differing quantities ${ }^{14}$. The latest reports on the role of EVs in intercellular communication underscore the stability of miRNAs in EVs and its usefulness as indicators of diverse processes ${ }^{15}$. Recently, the role of miRNA encapsulated in extracellular vesicles in cardiac remodeling upon stress has also been emphasized ${ }^{16}$.

Additionally, the selective nature of sorting of miRNA into EVs and the surface protein mediated specificity of EVs targeting to recipient cells ${ }^{17-19}$ prompt us to look for existence of differences in miRNA expression in both plasma and EVs between ALL survivors treated with doxorubicin and healthy controls. Here, we test the hypothesis that doxorubicin-induced tissue injury is responsible for dysregulation of the transcriptional network, which drives long-term side effects of anthracyclines and which manifests in altered circulating miRNA expression.

\section{Results}

\section{Characteristics of the studied groups}

There was no statistically significant difference in sex and there was a borderline nonsignificant difference in age between the 66 ALL patients and 61 healthy blood donors. Subsequently, we used the results of complete blood count and lipid panel tests to compare the two study groups (as presented in Table 1). Additionally, for ALL cohort, we collected echocardiographic data, which as expected remain within the normal range. Detailed information about echocardiographic parameters of ALL patients was presented in our previous publication ${ }^{20}$.

Table 1. Basic characteristics of the study groups. The median (range) values are reported; the p-value corresponds to the Fisher's exact test or Wilcoxon signed-rank test for equality of location parameters in the two groups.

\begin{tabular}{|c|c|c|c|}
\hline & Controls $(\mathbf{n = 6 1})$ & ALL $(\mathbf{n}=66)$ & p-value \\
\hline Sex, F/M ratio & 1.34 & 1.35 & 0.981 \\
\hline Age, years & $23(18-42)$ & $22(18-38)$ & 0.056 \\
\hline Creatinine $(\boldsymbol{\mu m o l} / \mathbf{l})$ & $\mathbf{7 6}(\mathbf{4 8 - 1 1 8})$ & $\mathbf{6 8}(\mathbf{4 2 - 9 7})$ & $\mathbf{9 . 0 E - 3}$ \\
\hline Total cholesterol $(\mathbf{m m o l} / \mathbf{l})$ & $4.50(2.49-6.60)$ & $4.2(3.1-5.9)$ & 0.146 \\
\hline HDL $(\mathbf{m m o l} / \mathbf{l})$ & $1.71(0.93-2.66)$ & $1.67(0.93-2.79)$ & 0.362 \\
\hline LDL $(\mathbf{m m o l} / \mathbf{l})$ & $\mathbf{2 . 6 9}(\mathbf{1 . 0 2}-\mathbf{4 . 8 9})$ & $\mathbf{2 . 2}(\mathbf{1 . 2}-\mathbf{4 . 1})$ & $\mathbf{1 . 5 E - 4}$ \\
\hline
\end{tabular}




\begin{tabular}{|c|c|c|c|}
\hline Triglycerides(mmol/l) & $0.94(0.31-3.49)$ & $0.80(0.34-2.40)$ & $1.4 \mathrm{E}-3$ \\
\hline RBC (10/ $\mu \mathrm{l})$ & $4.95(4.06-5.92)$ & $4.98(4.02-5.68)$ & 0.877 \\
\hline Hemoglobin (g/dl) & $14.1(12.1-17.1)$ & $14.95(12.50-16.90)$ & 0.100 \\
\hline Hematocrit (\%) & $42.5(37.0-51.1)$ & $43.5(36.0-47.7)$ & 0.794 \\
\hline $\operatorname{MCV}$ (fl) & $87.1(75.5-94.1)$ & $87.4(79.0-94.3)$ & 0.639 \\
\hline MCH (pg) & $29.2(24.4-31.8)$ & $29.9(26.2-33.2)$ & 2.0E-03 \\
\hline MCHC (g/dl) & $33.3(31.3-35.3)$ & $34.4(32.2-36.5)$ & 1.8E-08 \\
\hline Platelets $\left(10^{3} / \mu \mathrm{l}\right)$ & $321(147-377)$ & $266(168-415)$ & 0.547 \\
\hline MPV (fl) & $8.0(4.3-10.1)$ & $10.7(8.8-12.7)$ & $<2.2 \mathrm{E}-16$ \\
\hline White blood cells $\left(10^{3} / \mu \mathrm{l}\right)$ & $5.88(4.04-11.30)$ & $5.48(2.64-13.04)$ & 0.426 \\
\hline Neutrophils $\left(10^{3} / \mu \mathrm{l}\right)$ & $3.40(1.83-7.00)$ & $3.05(1.20-12.20)$ & 0.483 \\
\hline Lymphocytes $\left(10^{3} / \mu \mathrm{l}\right)$ & $1.7(1.1-2.7)$ & $1.6(0.3-3.2)$ & 0.066 \\
\hline Monocytes $\left(10^{3} / \mu \mathrm{l}\right)$ & $0.5(0.22-1.00)$ & $0.5(0.2-1.1)$ & 0.516 \\
\hline Eosinophils $\left(10^{3} / \mu \mathrm{l}\right)$ & $0.1(0.0-0.7)$ & $0.1(0.0-0.5)$ & 0.090 \\
\hline Basophils $\left(10^{3} / \mu \mathrm{l}\right)$ & $0.0(0.0-0.1)$ & $0.0(0.0-0.1)$ & 0.010 \\
\hline
\end{tabular}

HDL - high-density lipoproteins; LDL - low-density lipoproteins; RBC - red blood cells; MCV - mean; corpuscular volume; MCH - mean corpuscular hemoglobin; MCHC - mean corpuscular hemoglobin concentration; MPV - mean platelet volume

\section{$\underline{\text { Extracellular vesicles characteristics }}$}

Nanoparticle Tracking Analysis (NTA) analysis revealed that the median vesicle size was $69.75 \mu \mathrm{m}$ (65.05-73.35) and their concentration was $9.17 \mathrm{E}+12$ particles $/ \mathrm{ml}(5.58 \mathrm{E}+12-1.46 \mathrm{E}+13)$. There were no significant differences between control and ALL groups in particle size and concentration as Wilcoxon signed-rank test indicated.

\section{Differential expression of miRNAs in blood plasma and exosomes}

The miRNA sequencing was performed in 61 patients with ALL and 59 control subjects. Due to insufficient RNA amount, 7 samples were excluded from analysis. After removal of unexpressed miRNAs, 1986 miRNAs-precursor pairs were identified in plasma samples and EVs together. To better understand the role of miRNAs in ALL patients, we decided to compare miRNA expression in plasma and EVs separately. The comparison of ALL cases and controls allowed to detection of 201 miRNAs in plasma (Supplementary Material 1) and 49 miRNAs in EVs (Supplementary Material 2). The top 10 miRNAs differentially expressed in plasma and EVs between control and ALL survivors are shown in Table 2 in the top and middle panels, respectively. Only 2 miRNAs with reduced expression (miR-500a and miR-500b) were common to blood plasma and EVs (Figure $1)$. 


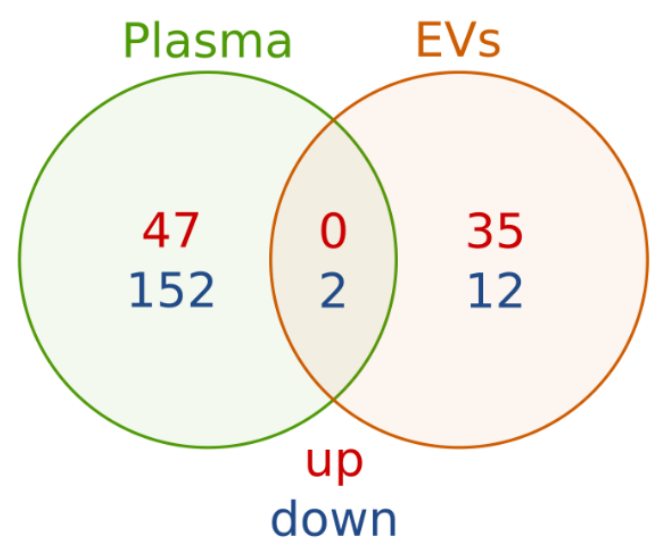

Figure 1. Venn diagram of differentially expressed miRNAs between ALL patients and control in plasma, EVs. The red color indicates up-regulated miRNAs, whereas the blue color indicates the down-regulated miRNAs.

Additionally, we looked whether miRNAs can be differentially distributed between the plasma and EVs in ALL survivors with respect to controls. This analysis shows that particular miRNAs in ALL survivors are preferentially enriched in plasma or EVs compartment in comparison to healthy controls. We discovered 95 miRNAs, of which 73 were plasma-specific $(\operatorname{logFC}>0)$ and 22 were EVs-specific $(\log \mathrm{FC}<0)($ Supplementary Material 3). Table 2, bottom panel, shows the top 10 results for differentially expressed miRNAs in blood plasma and EVs as well as miRNAs differentially distributed between these compartments.

Table 2. The top 10 differentially expressed miRNAs between controls and ALL survivors in blood plasma (top panel), EVs (middle panel) and differentially distributed between these compartments (bottom panel).

\begin{tabular}{|c|c|c|c|c|c|}
\hline \multicolumn{7}{|c|}{ Plasma } \\
\hline miRNA & Precursor & logFC & logCPM & p-value & FDR \\
\hline miR-184 & mir-184 & -5.358 & 8.897 & $2.49 \mathrm{E}-16$ & $4.95 \mathrm{E}-13$ \\
\hline miR-324-5p & mir-324 & -3.276 & 1.897 & $4.62 \mathrm{E}-10$ & $4.59 \mathrm{E}-07$ \\
\hline miR-4753-5p & mir-4753 & -3.149 & 1.352 & $2.08 \mathrm{E}-09$ & $1.05 \mathrm{E}-06$ \\
\hline let-7g-5p & let-7g & -0.701 & 13.642 & $2.11 \mathrm{E}-09$ & $1.05 \mathrm{E}-06$ \\
\hline miR-579-5p & mir-579 & -3.385 & 1.968 & $4.17 \mathrm{E}-09$ & $1.66 \mathrm{E}-06$ \\
\hline miR-1-3p & mir-1-2 & -2.993 & 7.536 & $6.95 \mathrm{E}-09$ & $2.16 \mathrm{E}-06$ \\
\hline miR-1-3p & mir-1-1 & -2.973 & 7.480 & $7.61 \mathrm{E}-09$ & $2.16 \mathrm{E}-06$ \\
\hline miR-3140-3p & mir-3140 & -3.108 & 1.629 & $1.88 \mathrm{E}-08$ & $4.67 \mathrm{E}-06$ \\
\hline miR-3939 & mir-3939 & -2.335 & 1.270 & $2.82 \mathrm{E}-07$ & $6.21 \mathrm{E}-05$ \\
\hline miR-1273c & mir-1273c & 4.799 & 3.364 & $4.13 \mathrm{E}-07$ & $8.21 \mathrm{E}-05$ \\
\hline
\end{tabular}




\begin{tabular}{|c|c|c|c|c|c|}
\hline \multicolumn{7}{|c|}{ EVs } \\
\hline miRNA & Precursor & logFC & logCPM & p-value & FDR \\
\hline miR-221-5p & mir-221 & 3.766 & 4.657 & $3.26 \mathrm{E}-11$ & $6.48 \mathrm{E}-08$ \\
\hline miR-199a-3p & mir-199a-2 & 1.722 & 9.299 & $9.30 \mathrm{E}-10$ & $4.63 \mathrm{E}-07$ \\
\hline miR-199b-3p & mir-199b & 1.722 & 9.298 & $9.31 \mathrm{E}-10$ & $4.63 \mathrm{E}-07$ \\
\hline miR-199a-3p & mir-199a-1 & 1.722 & 9.298 & $9.32 \mathrm{E}-10$ & $4.63 \mathrm{E}-07$ \\
\hline miR-203a-3p & mir-203a & 3.924 & 5.776 & $1.45 \mathrm{E}-09$ & $5.77 \mathrm{E}-07$ \\
\hline miR-574-5p & mir-574 & 3.101 & 3.979 & $1.48 \mathrm{E}-08$ & $4.89 \mathrm{E}-06$ \\
\hline miR-148a-5p & mir-148a & -2.639 & 5.911 & $2.00 \mathrm{E}-08$ & $5.68 \mathrm{E}-06$ \\
\hline miR-200a-3p & mir-200a & 2.288 & 8.178 & $2.78 \mathrm{E}-08$ & $6.89 \mathrm{E}-06$ \\
\hline miR-145-5p & mir-145 & 6.219 & 4.239 & $3.27 \mathrm{E}-08$ & $7.21 \mathrm{E}-06$ \\
\hline miR-378i & mir-378i & 1.891 & 6.074 & $1.07 \mathrm{E}-07$ & $2.13 \mathrm{E}-05$ \\
\hline \multicolumn{7}{|c|}{ Differentially distributed between plasma and EVs } & \\
\hline miRNA & Precursor & logFC & logCPM & p-value & FDR \\
\hline miR-184 & mir-184 & -6.218 & 8.897 & $7.60 \mathrm{E}-14$ & $1.51 \mathrm{E}-10$ \\
\hline miR-1-3p & mir-1-2 & -5.072 & 7.536 & $1.19 \mathrm{E}-12$ & $1.18 \mathrm{E}-09$ \\
\hline miR-1-3p & mir-1-1 & -4.935 & 7.480 & $3.52 \mathrm{E}-12$ & $2.33 \mathrm{E}-09$ \\
\hline miR-548am-5p & mir-548am & 7.756 & 3.824 & $3.68 \mathrm{E}-08$ & $1.75 \mathrm{E}-05$ \\
\hline miR-548o-5p & mir-548o-2 & 7.625 & 3.824 & $5.56 \mathrm{E}-08$ & $1.75 \mathrm{E}-05$ \\
\hline miR-548c-5p & mir-548c & 7.625 & 3.824 & $5.58 \mathrm{E}-08$ & $1.75 \mathrm{E}-05$ \\
\hline miR-208b-3p & mir-208b & 7.869 & 1.993 & $6.18 \mathrm{E}-08$ & $1.75 \mathrm{E}-05$ \\
\hline miR-199b-3p & mir-199b & -1.982 & 9.298 & $4.26 \mathrm{E}-07$ & $9.02 \mathrm{E}-05$ \\
\hline miR-199a-3p & mir-199a-1 & -1.982 & 9.298 & $4.26 \mathrm{E}-07$ & $9.02 \mathrm{E}-05$ \\
\hline miR-199a-3p & mir-199a-2 & -1.977 & 9.299 & $4.54 \mathrm{E}-07$ & $9.02 \mathrm{E}-05$ \\
\hline
\end{tabular}

$\operatorname{logFC}$ - log fold change, $\log \mathrm{CPM}$ - log counts per million, FDR - false discovery rate

\section{KEGG and GO enrichment analysis}

To gain insight into the potential functional role of global miRNA expression changes, we performed KEGG pathway analysis for target genes of significant miRNAs in plasma, EVs, and differential distribution analysis, taking into account the magnitude of change between tested conditions $(\log F C)$. The top 15 enriched KEGG terms of this analysis are shown in Figure 2. Details of all significant KEGG pathways can be found in Supplementary Material 4. Not surprisingly, a higher number of enriched terms were found for the plasma set, as more differentially expressed miRNAs were identified. Among the 15 most prominent pathways in each case, many were related to or strongly associated with cardiomyopathies, such as 'axon guidance, 'MAPK', 'ErbB', 'regulation of actin cytoskeleton' and 'neurotrophin 'signaling. Interestingly, these have previously been associated with cardiac function or the effects of anthracyclines' actions. However, the term 'dilated cardiomyopathy' appeared only in the KEGG analysis of EVs (Figure 2B). Whereas dilated cardiomyopathy, as well as arrhythmogenic right ventricular cardiomyopathy terms, are present in 
the analysis, which corresponds to miRNA differentially distributed between plasma and EVs in cancer survivors with respect to controls (Figure 2C). This supports the hypothesis that anticancer therapy-initiated processes may influence miRNA secretion to the extracellular environment and that miRNA in these two compartments may have a different role and target cells. Moreover, alterations in miRNA secretion to the extracellular environment may have a particularly significant role in the development of cardiac complications.
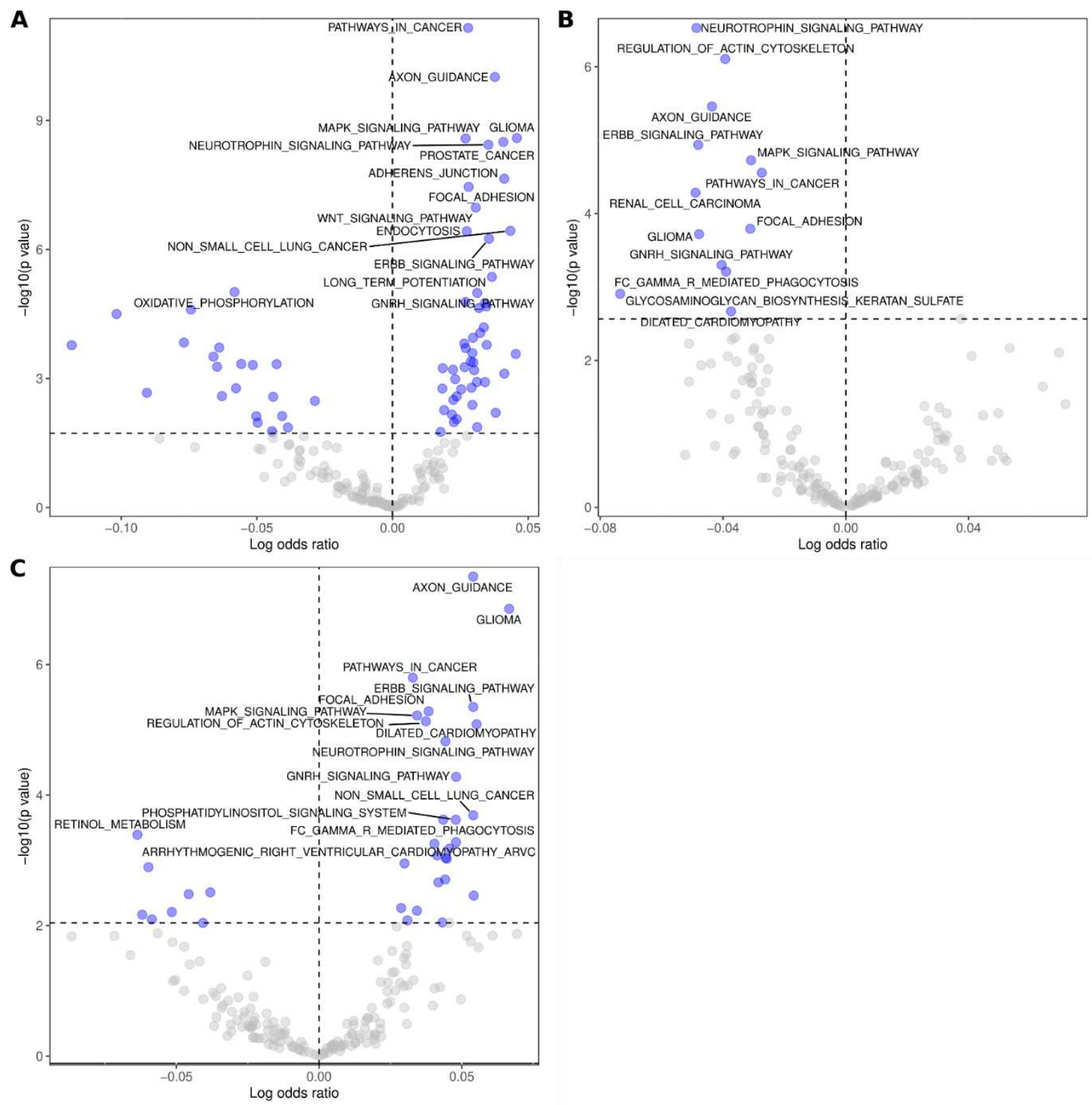

Figure 2. Results of the KEGG enrichment analysis among the targets of differentially expressed miRNAs in plasma (A), extracellular vesicles (B), and between the compartments

$\underline{(\mathbf{C})}$. 
Next, for the three sets of miRNAs defined above, we performed GO term enrichment analysis, for up- and down-regulated miRNAs separately. For plasma, we detected 212 and 93 enriched terms for up- and down-regulated miRNAs, respectively, while for a set of EVs, we detected 195 and 127 enriched terms, respectively (Supplementary Material 5). As for differentially distributed miRNAs, we detected 123 terms for upregulated miRNAs, which represent plasma-specific distribution, whereas 181 terms for downregulated miRNAs represent EVs-specific distribution (Supplementary Material 5).

The top 15 GO terms for each gene set were presented on Figure 3. Among plasma up- and EVs down-regulated GO terms 'muscle tissue development' and 'striated muscle tissue development' are present. 'Cardiac muscle tissue development' and 'cellular response to transforming growth factor beta stimulus', 'striated muscle cell proliferation' and 'heart morphogenesis' terms are unique for EVs "down-regulated" GO. Moreover, when all significant GO were filtered against "cardiac" term, ontologies such as 'cardiac muscle cell action potential', 'cardiac muscle cell contraction', 'cardiac muscle hypertrophy in response to stress' or 'cardiac muscle adaptation' appeared. 


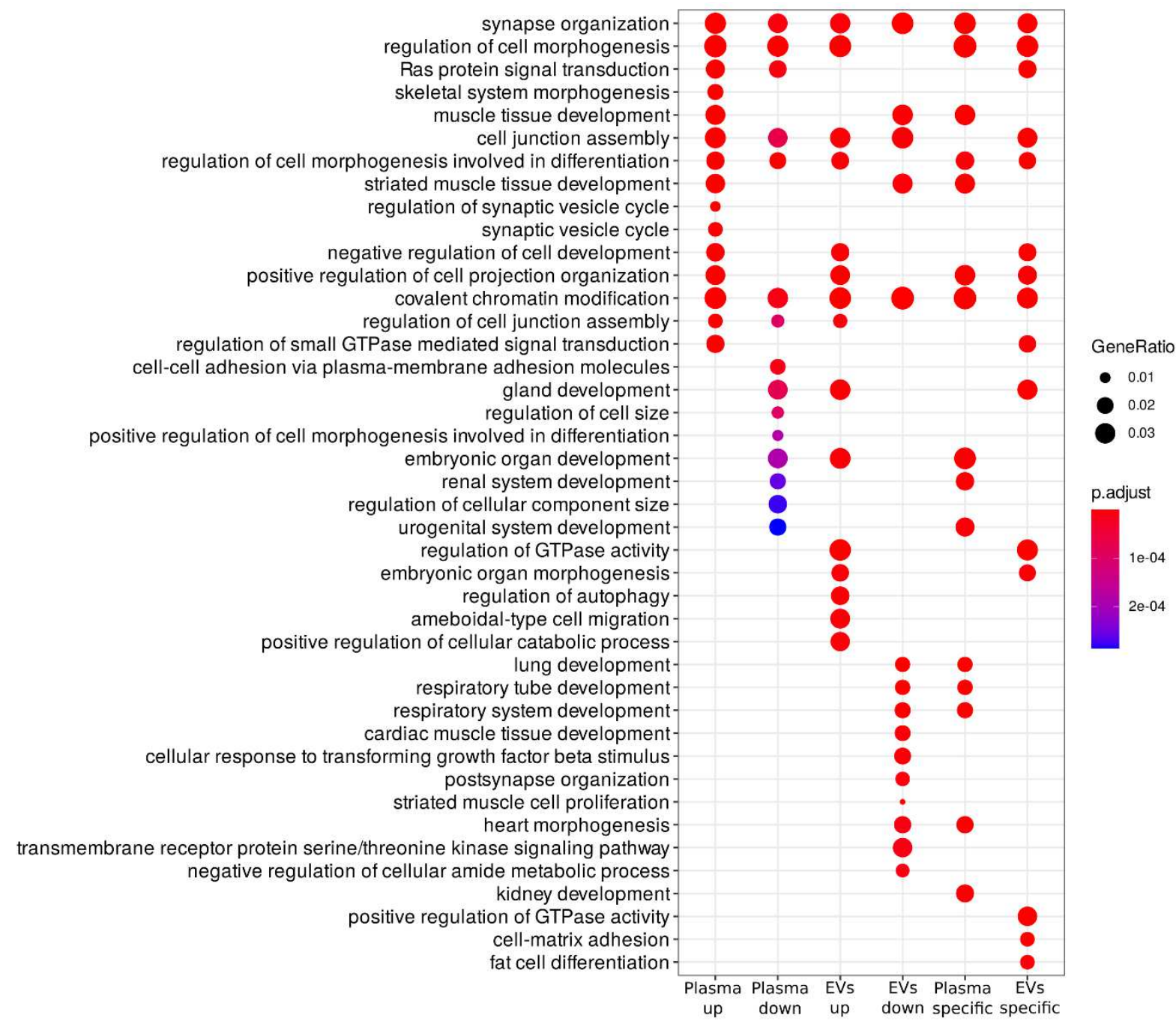

Figure 3. Results of the GO enrichment analysis (top 15 terms for each group) among targets of differentially expressed miRNAs in plasma, extracellular vesicles, and between the two compartments (plasma up - analysis for miRNA up-regulated in plasma, plasma down - analysis for miRNA down-regulated in plasma, EVs up - analysis for miRNA up-regulated in EVs, EVs down - analysis for miRNA down-regulated in EVs, plasma specific - analysis for miRNA that abundance in plasma is higher than in EVs in comparison to controls, EVs specific - analysis for miRNA that abundance in EVs is higher than in plasma in comparison to controls ).

Finally, we looked upon the "hallmark" gene sets from the Molecular Signature Database (MSigDB), to identify the relevant biological processes that are regulated by the target genes of our miRNAs sets. Briefly, our results are presented in Figure 4. Detailed information about discovered hallmarks are in Supplementary Material 6. 'Response to UV' is present in all analyses except plasma down-regulated miRNAs. For plasma up- and EVs down-regulated miRNAs, we found 
terms related to cell division/DNA damage ('G2M checkpoint, 'mitotic spindle'). 'TNFA via NFKB' is present for plasma down-regulated miRNA and for EVs up-regulated, it is also enriched in EVs of ALL survivors in comparison to plasma ('EVs specific'). Epithelial-mesenchymal transition is present for EVs up-regulated, plasma specific and EVs specific. TGF $\beta$ signaling is present for EVs up- and down-regulated miRNA as well as for miRNA with altered distribution between plasma and EVs in comparison to healthy controls. 'Hypoxia' and 'apoptosis' terms are unique for these miRNAs which are enriched in plasma when compared to EVs in ALL survivors (plasma specific). 'NOTCH signaling' is specific for EVs up, plasma down and plasma specific miRNA sets.

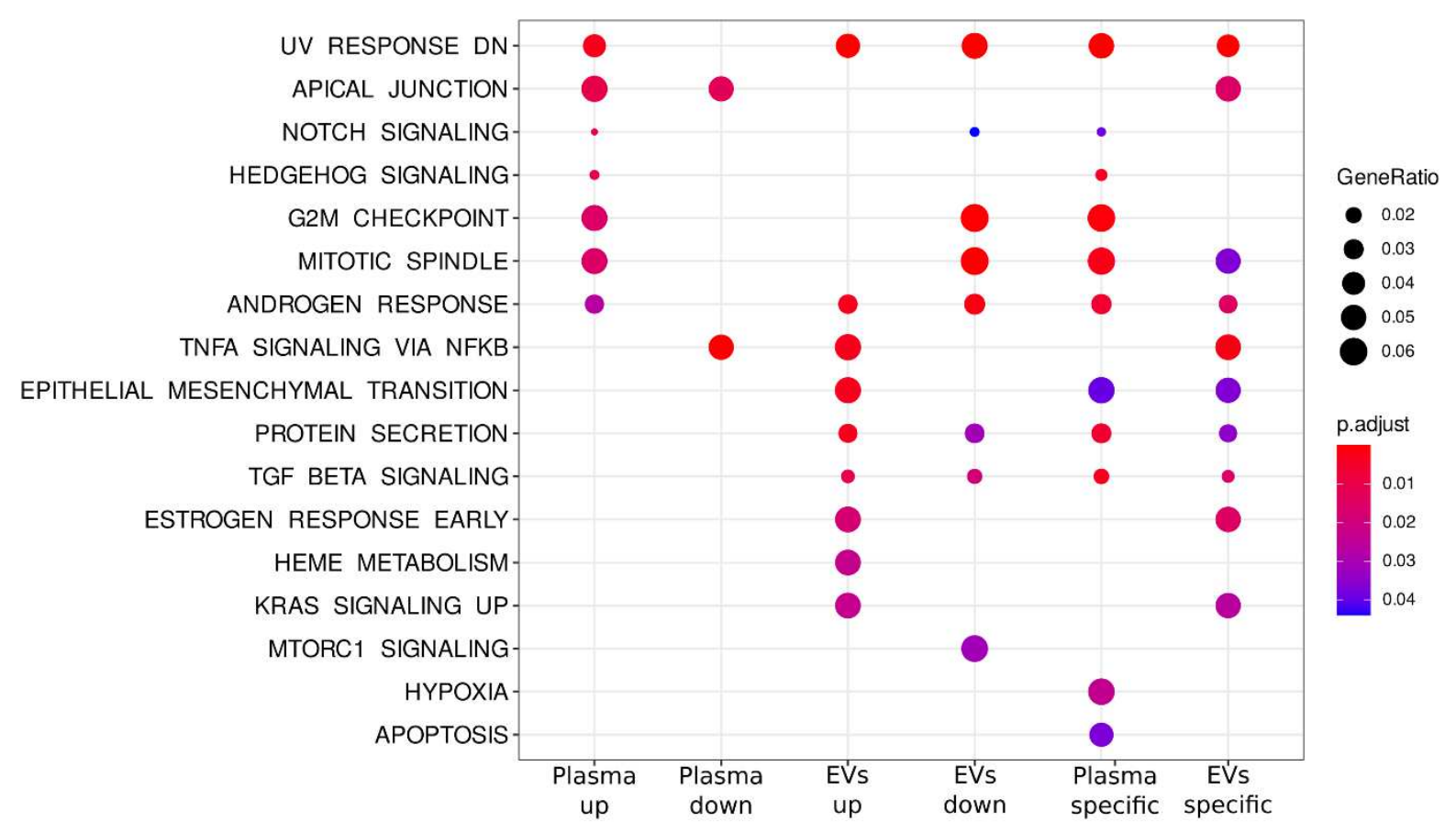

Figure 4. Results of the 'hallmark' enrichment analysis among targets of differentially expressed miRNAs in plasma, extracellular vesicles, and between the two compartments (plasma up - analysis for miRNA up-regulated in plasma, plasma down - analysis for miRNA downregulated in plasma, EVs up - analysis for miRNA up-regulated in EVs, EVs down - analysis for miRNA down-regulated in EVs, plasma specific - analysis for miRNA that abundance in plasma is higher than in EVs in comparison to controls, EVs specific - analysis for miRNA that abundance in EVs is higher than in plasma in comparison to controls ).:

Altogether, these results show that both miRNA compartments, vesicular and of total plasma, point to processes that may led to cardiomyopathy development, including TGF $\beta$ signaling, EMT and contraction-related issues. However, pathways regulated by differentially expressed miRNAs 
contained within two compartments are different and thus may diversely contribute to cardiac complications. Additionally, the analysis of differentially distributed miRNAs implies that some of miRNA secretory mechanisms might be dysregulated in ALL survivors.

\section{miRNAs associated with cardiomyopathy}

With data on transcriptomic alterations in former ALL patients, we asked whether our differentially expressed miRNAs are unique for such group or despite different cardiac disease origins share common features with patients suffering from clinically manifested cardiomyopathies. To achieve that, we used as a validation cohort data of Akat et $\mathrm{al}^{13}$ which includes samples from people with idiopathic cardiomyopathy (ICM) or dilated cardiomyopathy (DCM). We re-analyzed this data set and compared the differentially expressed miRNAs in plasma and EVs sets from our data with miRNAs differentially expressed between the serum of healthy individuals and patients with ICM or DCM. We found that 14 and 13 differentially expressed miRNAs in the plasma of ALL patients were also present in the blood plasma of ICM and DCM patients, respectively (Table 3). Similarly, we found 14 and 8 differentially expressed miRNAs in EVs of ALL patients that were also presented in the blood plasma of ICM and DCM patients, respectively (Table 3). These results suggest that despite echocardiographic measurements did not reveal significant pathological functional changes in relatively young ALL survivors, particular miRNAs may be, even at such early point, a good indicator of molecular processes leading later to cardiomyopathy.

Table 3. List of differentially expressed miRNAs in plasma or EVs of ALL patients shared with ICM or DCM datasets.

\begin{tabular}{|c|c|}
\hline \multicolumn{1}{|c|}{ Group } & Common DE miRNA \\
\hline ICM and ALL plasma & $\begin{array}{c}\text { miR-208b, miR-3680, miR-202, miR-101, miR-769, miR-511, miR-181b, } \\
\text { miR-216a, miR-210, miR-3158, miR-584, miR-455, miR-95, miR-1277 }\end{array}$ \\
\hline $\begin{array}{c}\text { DCM and ALL } \\
\text { plasma }\end{array}$ & $\begin{array}{r}\text { miR-1, miR-208b, miR-144, miR-194, miR-511, miR-181b, miR-216a, miR- } \\
\text { 210, miR-3158, miR-584, miR-455, miR-193a, miR-95 }\end{array}$ \\
\hline ICM and ALL EVs & $\begin{array}{c}\text { miR-199b, miR-148a, miR-200a, miR-361, miR-429, miR-21, miR-132, miR- } \\
\text { 15b, miR-215, miR-200b, miR-197, miR-10b, miR-29a, miR-143 }\end{array}$ \\
\hline
\end{tabular}




\section{miRNAs and echocardiographic parameters}

In differential miRNA analysis, we obtained multiple results that suggest the association of circulating miRNAs in plasma as well as in EVs with cardiomyopathy. Therefore, we asked whether DE miRNAs are related to cardiac system functioning. To answer that question, we correlate the DE miRNA from each compartment with echocardiographic parameters (Supplementary Material 7 and Supplementary Material 8). We found that many of DE miRNAs correlate with indicators of cardiac function. However, it is hard to say which of them are the most effective predictors and which of compartments: the plasma or EVs is more informative. Therefore, we decided to test the expression of most variable miRNAs with echocardiographic parameters, in each compartment separately. First, we filter out all miRNAs with a median expression of 5 pseudocounts in each compartment. Then, using Levene's test, we selected significant miRNAs $($ FDR $<0.05)$ that differ between ALL survivors and controls (Table 4), in plasma and EVs separately.

Table 4. Results of the Levene's test for equality of variances in plasma and EVs between ALL survivors and controls.

\begin{tabular}{|c|c|c|c|}
\hline \multicolumn{5}{|c|}{ Plasma } \\
\hline miRNA & Precursor & p-value & FDR \\
\hline miR-423-3p & mir-423 & $1.43 \mathrm{E}-07$ & $4.85 \mathrm{E}-05$ \\
\hline miR-144-3p & mir-144 & $1.11 \mathrm{E}-06$ & $1.88 \mathrm{E}-04$ \\
\hline let-7g-5p & mir-25 & $5.62 \mathrm{E}-06$ & $6.33 \mathrm{E}-04$ \\
\hline miR-101-3p & let-7g & $2.50 \mathrm{E}-05$ & $2.01 \mathrm{E}-03$ \\
\hline miR-101-3p & mir-101-2 & $3.53 \mathrm{E}-05$ & $2.01 \mathrm{E}-03$ \\
\hline miR-342-5p & mir-342 & $3.56 \mathrm{E}-05$ & $2.01 \mathrm{E}-03$ \\
\hline miR-501-3p & mir-501 & $9.09 \mathrm{E}-05$ & $4.39 \mathrm{E}-03$ \\
\hline miR-532-5p & mir-532 & $2.32 \mathrm{E}-04$ & $9.80 \mathrm{E}-03$ \\
\hline miR-16-2-3p & mir-16-2 & $3.80 \mathrm{E}-04$ & $1.43 \mathrm{E}-02$ \\
\hline miR-140-3p & mir-140 & $6.00 \mathrm{E}-04$ & $1.69 \mathrm{E}-02$ \\
\hline miR-182-5p & mir-182 & $6.68 \mathrm{E}-04$ & $1.69 \mathrm{E}-02$ \\
\hline miR-486-5p & mir-486-1 & $6.95 \mathrm{E}-04$ & $1.69 \mathrm{E}-02$ \\
\hline miR-486-5p & mir-486-2 & $7.00 \mathrm{E}-04$ & $1.69 \mathrm{E}-02$ \\
\hline miR-215-5p & mir-215 & $1.52 \mathrm{E}-03$ & $1.69 \mathrm{E}-02$ \\
\hline miR-1180-3p & mir-1180 & $1.78 \mathrm{E}-03$ & $3.42 \mathrm{E}-02$ \\
\hline let-7a-5p & let-7a-3 & $2.09 \mathrm{E}-03$ & $3.72 \mathrm{E}-02$ \\
\hline
\end{tabular}




\begin{tabular}{|c|c|c|c|}
\hline let-7a-5p & let-7a-2 & $2.09 \mathrm{E}-03$ & $3.72 \mathrm{E}-02$ \\
\hline let-7a-5p & let-7a-1 & $2.09 \mathrm{E}-03$ & $3.72 \mathrm{E}-02$ \\
\hline miR-100-5p & mir-100 & $2.24 \mathrm{E}-03$ & $3.79 \mathrm{E}-02$ \\
\hline let-7c-5p & let-7c & $2.82 \mathrm{E}-03$ & 4.54E-02 \\
\hline \multicolumn{4}{|c|}{ EVs } \\
\hline miRNA & Precursor & p-value & FDR \\
\hline miR-144-3p & mir-144 & $2.73 \mathrm{E}-06$ & $1.07 \mathrm{E}-03$ \\
\hline miR-7976 & mir-7976 & $5.55 \mathrm{E}-06$ & $1.09 \mathrm{E}-03$ \\
\hline miR-6747-3p & mir-6747 & $2.11 \mathrm{E}-05$ & $2.76 \mathrm{E}-03$ \\
\hline let-7f-5p & let-7f-1 & $4.38 \mathrm{E}-05$ & $2.85 \mathrm{E}-03$ \\
\hline let-7a-5p & let-7a-3 & $5.07 \mathrm{E}-05$ & $2.85 \mathrm{E}-03$ \\
\hline let-7a-5p & let-7a-1 & $5.09 \mathrm{E}-05$ & $2.85 \mathrm{E}-03$ \\
\hline let-7a-5p & let-7a-2 & $5.09 \mathrm{E}-05$ & $2.85 \mathrm{E}-03$ \\
\hline let-7f-5p & let-7f-2 & $6.73 \mathrm{E}-05$ & $3.30 \mathrm{E}-03$ \\
\hline miR-486-5p & mir-486-1 & $2.02 \mathrm{E}-04$ & 7.99E-03 \\
\hline miR-486-5p & mir-486-2 & $2.04 \mathrm{E}-04$ & $7.99 \mathrm{E}-03$ \\
\hline let-7c-5p & let-7c & $3.63 \mathrm{E}-04$ & $1.22 \mathrm{E}-02$ \\
\hline miR-501-3p & mir-501 & $3.75 \mathrm{E}-04$ & $1.22 \mathrm{E}-02$ \\
\hline miR-26b-5p & mir-26b & 4.41E-04 & $1.33 \mathrm{E}-02$ \\
\hline miR-10b-5p & mir-10b & $7.17 \mathrm{E}-04$ & $2.01 \mathrm{E}-02$ \\
\hline miR-423-3p & mir-423 & 8.79E-04 & $2.30 \mathrm{E}-02$ \\
\hline let-7g-5p & let-7g & 9.97E-04 & $2.44 \mathrm{E}-02$ \\
\hline miR-101-3p & mir-101-2 & $1.29 \mathrm{E}-03$ & $2.86 \mathrm{E}-02$ \\
\hline miR-197-3p & mir-197 & $1.32 \mathrm{E}-03$ & $2.86 \mathrm{E}-02$ \\
\hline miR-101-3p & mir-101-1 & $1.58 \mathrm{E}-03$ & $3.26 \mathrm{E}-02$ \\
\hline miR-877-5p & mir-877 & $1.77 \mathrm{E}-03$ & $3.47 \mathrm{E}-02$ \\
\hline miR-3613-5p & mir-3613 & $2.13 \mathrm{E}-03$ & $3.98 \mathrm{E}-02$ \\
\hline
\end{tabular}

$\operatorname{logFC}$ - log fold change, $\log \mathrm{CPM}$ - log counts per million, FDR - false discovery rate

Then, we correlated each echocardiographic parameter with those miRNAs. Results of these analyses are presented on Figure 5. Among plasma miRNA, mir-let-7g-5p is positively correlated with the highest number of echocardiographic parameters, including left ventricle dimensions. Among vesicular miRNA, mir-144-3p is correlated with the highest number of variables measured. 


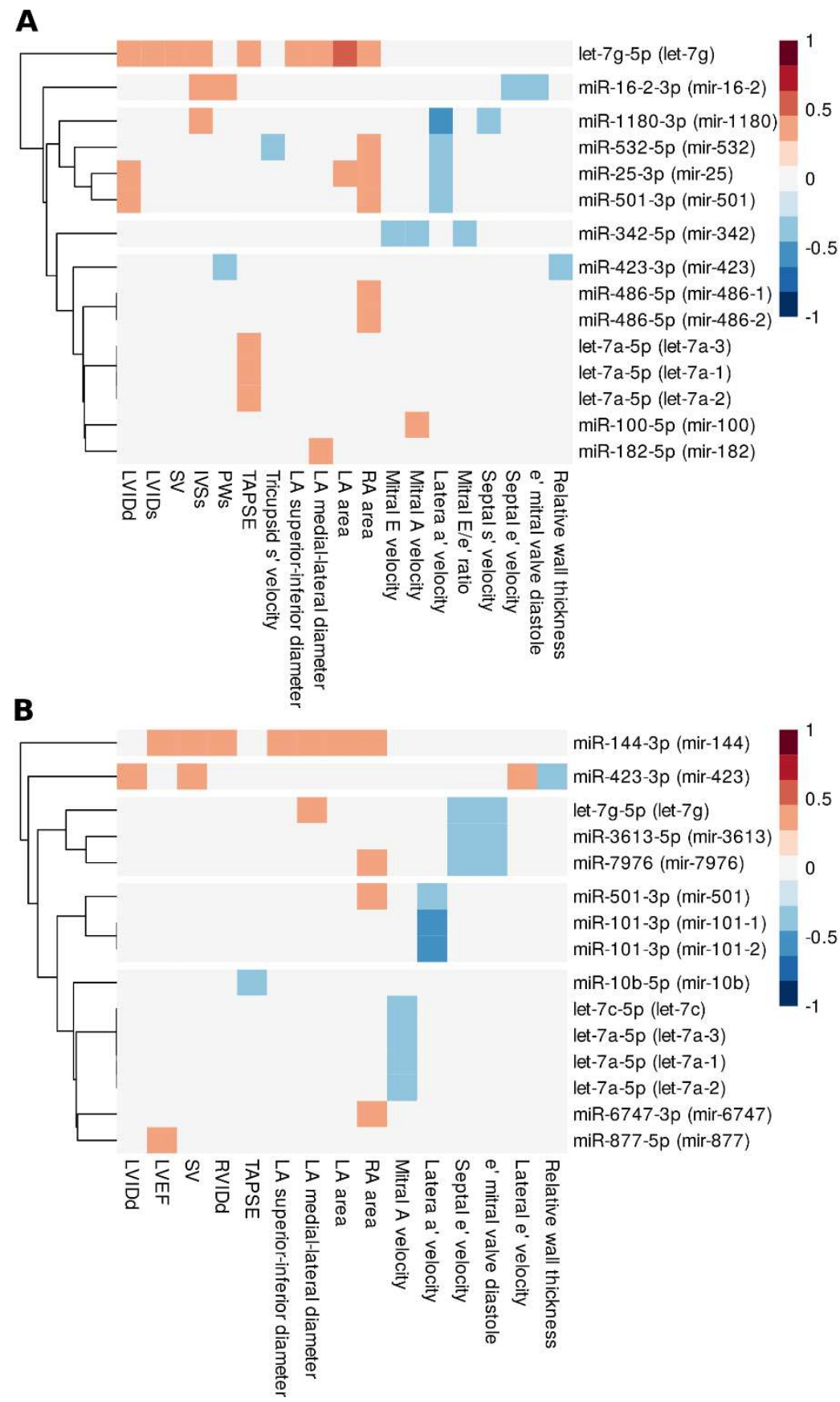

Figure 5. Correlation of the miRNAs, with dissimilar variance in plasma (A) and EVs (B) between control and ALL patients, with echocardiography parameters. The miRNAs without significant correlation were removed. Non-significant correlation was presented as white blocks. (LVID - left ventricular internal dimension in systole (s) or diastole (d), LVEF - left ventricular ejection fraction, SV - left ventricular stroke volume, IVSs - septal wall thickness, PWs - left ventricular 
posterior wall thickness, RVID - right ventricular internal dimension, TAPSE - tricuspid annular plane systolic excursion, LA area-left atrial area, RA - right atrial area)

\section{Discussion}

Here we present diverse lines of evidence that long-term molecular effects of doxorubicin action in ALL survivors include changes of miRNA abundance in circulation that may contribute to the development of cardiomyopathy, a major life-threatening long-term side effect of anthracycline treatment ${ }^{21,22}$. We sequenced miRNA to find those differentially expressed between ALL survivors and healthy subjects, characterized miRNA distribution between total plasma and extracellular vesicles, and used several bioinformatic tools to suggest processes active in these patients, Subsequently, we compared our findings with those previously described in cardiomyopathy patients. Finally, we searched for correlations between identified miRNA and discrete echocardiographic parameters, that may be suggestive of incipient cardiac dysfunction.

First, we confirmed that circulating miRNAs that are differentially expressed in ALL survivors in comparison to healthy people may indicate transcriptional alterations related to cardiac disease development.

KEGG enrichment analyses revealed that differentially expressed miRNA in EVs as well as miRNA that are differentially distributed between plasma and EVs are related to 'dilated cardiomyopathy' or 'arrhythmogenic cardiomyopathy'. Both morphological and functional changes in doxorubicininduced cardiomyopathy have been reported as similar to those of dilated cardiomyopathy. It involves presence of fibrotic areas, myofilaments loss with visible Z-discs disorganization. In advanced pathology, chambers dilation is present with concomitant reduction of ejection fraction and diastolic dysfunction ${ }^{23}$. We also found other KEGG terms significantly related to DCM, like 'ERBB signaling' which pathway plays a key role in maintaining cardiac structure ${ }^{24,25}$ as well as in restoring cardiac function after injury ${ }^{26}$. Its postnatal disruption leads to dilated cardiomyopathy ${ }^{27}$ and sensitizes heart to drug-induced toxicity ${ }^{28}$. In addition, molecular pathways related to cardiac rhythm and contraction are altered in ALL survivors, which is indicated by KEGG terms arrhythmogenic right ventricular cardiomyopathy', 'axon guidance' or 'neurotrophin signaling', which is essential for normal cardiac rhythm through the regulation of cardiac Ca2+ cycling ${ }^{29-31}$. This remains in line with reports showing that doxorubicin affects cardiac electrophysiological properties and may cause various type of arrhythmias ${ }^{23}$. Analysis of gene ontologies further supports this finding as among significant ontologies we identified such as related to heart, muscle, 
cardiocyte, cardiac structures (ventricle, valve), endocardial cushion and cardiac contraction and relaxation.

Our analyses of molecular pathways that are disturbed in ALL survivors revealed that differentially expressed miRNA are involved in the regulation of pathways related to DNA damage, which belongs to canonical effects of doxorubicin action involved in cardiac complications $^{8}$ and to pathological cardiac remodeling like NFK $\beta$ and TNF $\alpha$ signaling ${ }^{32}$. Among processes regulated specifically by miRNA differentially expressed in EVs we identified epithelial-to-mesenchymal- transition (EMT), process linked to therapytriggered fibrosis ${ }^{33,34}$ and senescence, which was described as a consequence of genotoxic treatment ${ }^{35,36}$ and was suggested to reinforce long-term cardiac complications of anticancer treatment $^{37}$.

Additionally, both this and GO analysis show that particularly miRNAs encapsulated in EVs are involved in TGF $\beta$ signaling. TGF $\beta$ is a master regulator of EMT ${ }^{38,39}$, which expression can be increased in the heart tissue many weeks after doxorubicin treatment ${ }^{40}$. It was shown that maintaining balance within this pathway is critical for cardiac contractile function, sarcomere kinetics, ion-channel gene expression, and cardiomyocyte survival $^{41,42}$.

Multiple of differentially expressed miRNAs in our study correlate with cardiac function parameters in ALL survivors, which supports the role of particular miRNAs in cardiac system functioning. However, due to redundancy of miRNA in transcriptomic network and interrelatedness between echocardiographic variables, it is difficult to identify miRNAs predictive of cardiac system function. Therefore, we used another approach, based on selecting the set of miRNAs that expression is most variable between groups in each of compartments. Strikingly, among plasma most variable miRNAs, let-7g-5p, correlated with the highest number of echocardiographic parameters, has been reported by Fu et al as involved in cardiac cells response to doxorubicin ${ }^{43}$.

We also show that similarities in miRNA expression between ALL survivors and patients with advanced, clinically manifested cardiomyopathies exist, despite that the patients in our studied group have not developed any significant cardiac phenotype yet, most probably due to young age and short time span between doxorubicin exposure and sample collection. 
Of the most variable miRNA set, miR-144, miR-10b and miR-101 are common for plasma and EVs in ALL survivors and ICM/DCM patients. miR-144-3p, having the highest statistical significance in EVs, is crucial for cardiac function as its loss worsened heart failure phenotype resulting with impaired late remodeling and decreased $\mathrm{LVEF}^{44}$. Moreover, mir-144 was identified as an important regulatory node in $\mathrm{DCM}^{45}$ and its expression was down-regulated both in samples from DCM patients and in a doxorubicininduced rodent model of cardiomyopathy ${ }^{46}$. Another study supporting important role of mir-144 shows that its loss resulted in ventricular dilation and impaired contractility, whereas intravenous delivery of this miRNA reduced infarcted area and improved cardiac function including LV fractional shortening, end-systolic volume, end-diastolic volume and ejection fraction ${ }^{47}$. Our study shows that miR-144-3p is positively correlated with the highest number of cardiac parameters including ejection fraction, a parameter that is used to define and to monitor the progress of anthracycline-induced cardiac disease ${ }^{48}$.

Interestingly, only vesicular expression of this miRNA is informative in terms of cardiac functioning. This phenomenon might be related to the specificity of both the EVs packaging and release as well as EVs uptake - being precise vesicular mir-144 might have either slightly different cellular origin or target than that circulating outside EVs. Abundance of RNA in extracellular space depends among others on the cellular system of RNA binding proteins (RBP) that are part of the cell cargo packing and exporting system, shown to be affected by doxorubicin ${ }^{49}$. E.g. doxorubicin changes expression of RBPs in rodent cardiomyocytes and in human induced pluripotent stem cell-derived cardiomyocytes. Additionally, our analyses reveal that especially miRNA that are differentially distributed between plasma and vesicles (i.e. miRNA that are more or less abundant in EVs than in plasma when compared to healthy people) in ALL survivors indicate processes related to cardiomyopathy. Of note, the term 'protein secretion' was present among enriched hallmarks. This supports the notion that changes in miRNA presence that we observe are closely linked to the alterations in RNA secretory mechanisms, possibly related to RNA binding proteins.

In summary, our study indicates that particular miRNA, including miR-144-3p and let-7g$5 \mathrm{p}$, could be considered as candidates for further studies on cardiac complications in 
doxorubicin treated cancer survivors. Moreover, we demonstrate that compartment that is studied as the source of miRNA should be carefully chosen, as the source of miRNA origin as well as its destination site may be different in the case of vesicular and total plasma fraction of miRNA. However, both compartments may be useful source of information on processes that in a long perspective can lead to cardiomyopathy development in former patients treated with anthracyclines, especially if we consider differential distribution between plasma and EVs.

We are aware that it would be of great value if such finding could be confirmed in the follow-up study at later time points in this population as well as validated in other populations of cancer survivors. The main study limitation is lack of verification of specificity of our findings, i.e. whether similar association between echocardiographic parameters and miRNA expression in blood exists also in healthy individuals or in various forms of cardiomyopathy.

\section{Materials and Methods}

\section{$\underline{\text { Study cohort }}$}

The survivor population was recruited from the Childhood Cancer Survivorship Clinic at the University Hospital in Kraków. Informed consent was obtained in accordance with the Declaration of Helsinki. The study was approved by the Bioethics Committee at the Jagiellonian University (Approval No. 122.6120.274.2015). Eligibility criteria included the following: (1) diagnosis of ALL before 18 years of age and (2) 5 or more years since the completion of cancer treatment (doxorubicin). Exclusion criteria included the following: (1) time from the end of therapy for ALL shorter than 5 years, (2) relapse or secondary cancer at the time of the study or during the 5 preceding years. The study participants underwent a comprehensive clinical evaluation, including a physical examination accompanied by anthropometric assessments. Healthy controls were recruited at the Blood Donation Center in Kraków, Poland. Blood sampling, biochemical analyses, and echocardiographic evaluation were performed as previously described ${ }^{20}$

\section{Isolation and characterization of EVs}

Plasma EVs were isolated with the miRCURY Exosome Isolation Kit (Exiqon, Qiagen, Aarhus, Denmark) according to the manufacturer's protocol. Size distribution of EVs was measured by Nanoparticle Tracking Analysis with NanoSight (Malvern Panalytical, Malvern, United Kingdom).

\section{RNA extraction and preparation of miRNA libraries}

Small RNA was extracted from EVs and total plasma with miRCURY RNA Isolation Kit (Exiqon, Qiagen). Libraries were prepared with NebNext Small RNA Library Prep (New England Biolabs, 
Ipswich, MA, USA). Quality control steps for libraries were performed on TapeStation (Agilent Technologies, Santa Clara, CA, USA) before and after size selection. cDNA concentration was measured using the Quantus fluorometer (Promega, Madison, Wisconsin, USA). Pooled libraries were sequenced with High Output v2.0 reagents on the NextSeq 500 sequencer (Illumina, San Diego, CA, USA).

\section{miRNA-seq analysis}

Demultiplexed sequenced reads from pooled libraries were quality checked with a FastQC software, v0.11.8[135]. The reads were then trimmed to remove primers and poor-quality bases with Cutadapt, v1.18[72]. Reads with length $<18$ or $>30$ nucleotides and reads without 3' adapter were removed. The cleaned reads were then aligned to miRBase database v22.1 [57] and counted using miRDeep2 software v0.0.8 [27]. Normalized miRNA read count generated from miRDeep2 was used in further differential expression analysis. The raw sequences, along with raw and normalized counts from miRDeep2 software were deposited in GEO (GSE145176).

\section{$\underline{\text { Statistical methods }}$}

All statistical analyses and filtering steps were performed in R (v3.5.2). Briefly, only miRNAs with expression in at least one sample were used in the statistical analysis. The differential expression was analyzed by the edgeR package with two different experimental designs. The first model (xp $\sim$ compartment + status:compartment) was used to test differences in miRNAs expression between ALL survivors and controls in plasma and EVs separately. The second model (xp status + compartment:status) was used to test whether miRNAs are differentially distributed between plasma and EVs in ALL survivors with respect to controls. Only results with FDR $<0.05$ were considered significant. The KEGG, GO, and hallmark enrichment analysis was performed with 'RbiomirGS' and 'clusterProfiler' R packages. Briefly, we used 'RbiomirGS' to find target genes for differentially expressed miRNAs in each analysis using various predictive algorithms from multiMIR's database v2.1. Then, we conduct a logistic regression-based gene set enrichment in 'RbiomirGS' to find significant KEGG terms. Since 'RbiomirGS' considers the change in expression ( $\log \mathrm{FC})$, for GO and hallmark enrichment analysis in 'clusterProfiler' we divided the significant results from each set on up and down-regulated miRNA. To keep GO analysis transparent, we only showed terms between levels 4 and 9. Only terms with FDR $<0.05$ were considered significant. The correlation between DE miRNA and echocardiographic parameters was tested with the 'Hmisc' package and then plotted with the 'pheatmap' package. To test in an unbiased fashion whether the expression in plasma/exosomes of miRNA correlates with selected echocardiographic parameters, we used normalized pseudocounts and Levene's test to select 
miRNAs that have the highest variance between cases and controls in plasma or EVs. For further analyses, we used only the ones which remain significantly differentially variable with FDR $<0.05$. We then performed correlation analysis with echocardiographic parameters similar to DE miRNAs.

\section{Conflict of interest: Authors report no conflict of interest}

\section{Acknowledgements}

The plasma miRNA expression data, from ICM and DCM patients, used for the analyses described in this manuscript were obtained from the Gene Expression Omnibus (GEO) database, www.ncbi.nlm.nih.gov/geo (accession no. GSE53081).

\section{Sources of funding}

This research was funded by the National Science Centre (Poland), grant No. 2015/17/D/NZ7/02165 (to J.T.-Ż.)

\section{Authors Contributions:}

JTZ, MTS, PW - contributed to conceptualization, wrote and edited this manuscript, MTS, PK, LD - performed bioinformatic and statistical analyses, MKW, EP - conducted experiments, JSG, BC, EN - contributed to conceptualization, provided samples, and collected clinical data, AS, TG - contributed to the conceptualization, All authors read and approved the final manuscript.

\section{Ethics approval and consent to participate}

The study was approved by the Bioethics Committee at the Jagiellonian University (approval No. 22.6120.274.2015).

\section{Data availability}

The raw RNA sequences, along with raw and normalized counts from miRDeep2 software were deposited in GEO (GSE145176).

\section{References}


1. Birch, J. M., Marsden, H. B., Jones, P. H., Pearson, D. \& Blair, V. Improvements in survival from childhood cancer: results of a population based survey over 30 years. Br Med J (Clin Res Ed) 296, 1372-1376 (1988).

2. Bonaventure, A. et al. Worldwide comparison of survival from childhood leukaemia for 1995-2009, by subtype, age, and sex (CONCORD-2): a population-based study of individual data for 89828 children from 198 registries in 53 countries. Lancet Haematol 4, e202-e217 (2017).

3. Lipshultz, S. E. et al. Late Cardiac Effects of Doxorubicin Therapy for Acute Lymphoblastic Leukemia in Childhood. http://dx.doi.org/10.1056/NEJM199103213241205 https://www.nejm.org/doi/10.1056/NEJM199103213241205?url_ver=Z39.882003\&rfr_id=ori\%3Arid\%3Acrossref.org\&rfr_dat=cr_pub\%3Dwww.ncbi.nlm.nih.gov (2010) doi:10.1056/NEJM199103213241205.

4. Jordan, J. H. et al. Left Ventricular Mass Change After Anthracycline Chemotherapy. Circ Heart Fail 11, e004560 (2018).

5. Octavia, Y. et al. Doxorubicin-induced cardiomyopathy: From molecular mechanisms to therapeutic strategies. Journal of Molecular and Cellular Cardiology 52, 1213-1225 (2012).

6. McLaughlin, D. et al. Signalling mechanisms underlying doxorubicin and Nox2 NADPH oxidase-induced cardiomyopathy: involvement of mitofusin-2. British Journal of Pharmacology 174, 3677-3695 (2017).

7. L'Ecuyer, T. et al. DNA damage is an early event in doxorubicin-induced cardiac myocyte death. American Journal of Physiology-Heart and Circulatory Physiology 291, H1273-H1280 (2006).

8. Qiao, X. et al. Uncoupling DNA damage from chromatin damage to detoxify doxorubicin. PNAS 117, 15182-15192 (2020).

9. Marinello, J., Delcuratolo, M. \& Capranico, G. Anthracyclines as Topoisomerase II Poisons: From Early Studies to New Perspectives. Int J Mol Sci 19, (2018).

10. Miller, K. D. et al. Cancer treatment and survivorship statistics, 2019. CA: A Cancer Journal for Clinicians 69, 363-385 (2019).

11. Stadiotti, l. et al. Arrhythmogenic cardiomyopathy: what blood can reveal? Heart Rhythm 16, 470-477 (2019).

12. Silverman, M. G. et al. Circulating miRNAs and Risk of Sudden Death in Patients With Coronary Heart Disease. JACC Clin Electrophysiol 6, 70-79 (2020).

13. Akat, K. M. et al. Comparative RNA-sequencing analysis of myocardial and circulating small RNAs in human heart failure and their utility as biomarkers. PNAS 111, 11151-11156 (2014).

14. Endzeliňš, E. et al. Detection of circulating miRNAs: comparative analysis of extracellular vesicle-incorporated miRNAs and cell-free miRNAs in whole plasma of prostate cancer patients. BMC Cancer 17, 730 (2017).

15. Kalani, M. Y. S. et al. Extracellular microRNAs in blood differentiate between ischaemic and haemorrhagic stroke subtypes. Journal of Extracellular Vesicles 9, 1713540 (2020).

16. Tian, C., Hu, G., Gao, L., Hackfort, B. T. \& Zucker, I. H. Extracellular vesicular MicroRNA-27a* contributes to cardiac hypertrophy in chronic heart failure. Journal of Molecular and Cellular Cardiology 143, 120-131 (2020).

17. Hoshino, A. et al. Tumour exosome integrins determine organotropic metastasis. Nature 527, 329-335 (2015).

18. Sancho-Albero, M. et al. Exosome origin determines cell targeting and the transfer of therapeutic nanoparticles towards target cells. J Nanobiotechnology 17, (2019).

19. Sanz-Rubio, D. et al. Stability of Circulating Exosomal miRNAs in Healthy Subjects. Sci Rep 8, 1-10 (2018). 
20. Sulicka-Grodzicka, J. et al. Cranial Irradiation in Childhood Acute Lymphoblastic Leukemia Is Related to Subclinical Left Ventricular Dysfunction and Reduced Large Artery Compliance in Cancer Survivors. J Clin Med 8, (2019).

21. Mertens, A. C. et al. Cause-Specific Late Mortality Among 5-Year Survivors of Childhood Cancer: The Childhood Cancer Survivor Study. J Natl Cancer Inst 100, 1368-1379 (2008).

22. Tukenova, M. et al. Role of Cancer Treatment in Long-Term Overall and Cardiovascular Mortality After Childhood Cancer. JCO 28, 1308-1315 (2010).

23. Benjanuwattra, J., Siri-Angkul, N., Chattipakorn, S. C. \& Chattipakorn, N. Doxorubicin and its proarrhythmic effects: A comprehensive review of the evidence from experimental and clinical studies. Pharmacological Research 151, 104542 (2020).

24. Lemmens Katrien, Doggen Kris \& De Keulenaer Gilles W. Role of Neuregulin-1/ErbB Signaling in Cardiovascular Physiology and Disease. Circulation 116, 954-960 (2007).

25. Rohrbach, S., Niemann, B., Silber, R.-E. \& Holtz, J. Neuregulin receptors erbB2 and erbB4 in failing human myocardium -- depressed expression and attenuated activation. Basic Res. Cardiol. 100, 240-249 (2005).

26. Galindo, C. L., Ryzhov, S. \& Sawyer, D. B. Neuregulin as a Heart Failure Therapy and Mediator of Reverse Remodeling. Curr Heart Fail Rep 11, 40-49 (2014).

27. Crone, S. A. et al. ErbB2 is essential in the prevention of dilated cardiomyopathy. Nat. Med. 8, 459-465 (2002).

28. Popat, S. \& Smith, I. E. Therapy Insight: anthracyclines and trastuzumab--the optimal management of cardiotoxic side effects. Nat Clin Pract Oncol 5, 324-335 (2008).

29. Feng, N. et al. Constitutive BDNF/TrkB signaling is required for normal cardiac contraction and relaxation. PNAS 112, 1880-1885 (2015).

30. Fulgenzi, G. et al. BDNF modulates heart contraction force and long-term homeostasis through truncated TrkB.T1 receptor activation. J Cell Biol 210, 1003-1012 (2015).

31. Miwa, K. et al. Axon Guidance of Sympathetic Neurons to Cardiomyocytes by Glial Cell LineDerived Neurotrophic Factor (GDNF). PLoS One 8, e65202 (2013).

32. Gordon, J. W., Shaw, J. A. \& Kirshenbaum, L. A. Multiple Facets of NF-kB in the Heart. Circulation Research 108, 1122-1132 (2011).

33. Kalluri, R. \& Neilson, E. G. Epithelial-mesenchymal transition and its implications for fibrosis. J Clin Invest 112, 1776-1784 (2003).

34. Sun, H. et al. Nucleolin protects against doxorubicin-induced cardiotoxicity via upregulating microRNA-21. J. Cell. Physiol. 233, 9516-9525 (2018).

35. Tato-Costa, J. et al. Therapy-Induced Cellular Senescence Induces Epithelial-toMesenchymal Transition and Increases Invasiveness in Rectal Cancer. Clin Colorectal Cancer 15, 170-178.e3 (2016).

36. Baar, M. P. et al. Targeted Apoptosis of Senescent Cells Restores Tissue Homeostasis in Response to Chemotoxicity and Aging. Cell 169, 132-147.e16 (2017).

37. Demaria, M. et al. Cellular Senescence Promotes Adverse Effects of Chemotherapy and Cancer Relapse. Cancer Discov 7, 165-176 (2017).

38. Zavadil, J., Cermak, L., Soto-Nieves, N. \& Böttinger, E. P. Integration of TGF-beta/Smad and Jagged1/Notch signalling in epithelial-to-mesenchymal transition. EMBO J. 23, 1155-1165 (2004).

39. Ferrarelli, L. K. Revisiting TGF- $\beta$ and EMT. Science 363, 941-943 (2019).

40. Mancilla, T. A., Andrews, T. \& Aune, G. Doxorubicin-Induced Chronic Upregulation of Transforming Growth Factor-beta. The FASEB Journal 30, 1205.6-1205.6 (2016).

41. Umbarkar, P. et al. Cardiomyocyte SMAD4-Dependent TGF- $\beta$ Signaling is Essential to Maintain Adult Heart Homeostasis. JACC: Basic to Translational Science 4, 41-53 (2019). 
42. Li, S. et al. Pro-oxidant effect of transforming growth factor- beta1 mediates contractile dysfunction in rat ventricular myocytes. Cardiovasc Res 77, 107-117 (2008).

43. Fu, J. et al. Let-7 $\mathrm{g}$ is involved in doxorubicin induced myocardial injury. Environ. Toxicol. Pharmacol. 33, 312-317 (2012).

44. He, Q. et al. Loss of miR-144 signaling interrupts extracellular matrix remodeling after myocardial infarction leading to worsened cardiac function. Sci Rep 8, 16886 (2018).

45. Huang, K. et al. Integrated Analysis of Hub Genes and miRNAs in Dilated Cardiomyopathy. BioMed Research International 2020, e8925420 (2020).

46. Tao, L., Yang, L., Huang, X., Hua, F. \& Yang, X. Reconstruction and Analysis of the IncRNAmiRNA-mRNA Network Based on Competitive Endogenous RNA Reveal Functional IncRNAs in Dilated Cardiomyopathy. Frontiers in Genetics 10, 1149 (2019).

47. Li, J. et al. Intravenous miR-144 reduces left ventricular remodeling after myocardial infarction. Basic Res Cardiol 113, 36 (2018).

48. Leerink, J. M. et al. Refining the 10-Year Prediction of Left Ventricular Systolic Dysfunction in Long-Term Survivors of Childhood Cancer. JACC: CardioOncology 3, 62-72 (2021).

49. Statello, L. et al. Identification of RNA-binding proteins in exosomes capable of interacting with different types of RNA: RBP-facilitated transport of RNAs into exosomes. PLOS ONE 13, e0195969 (2018). 
Figures

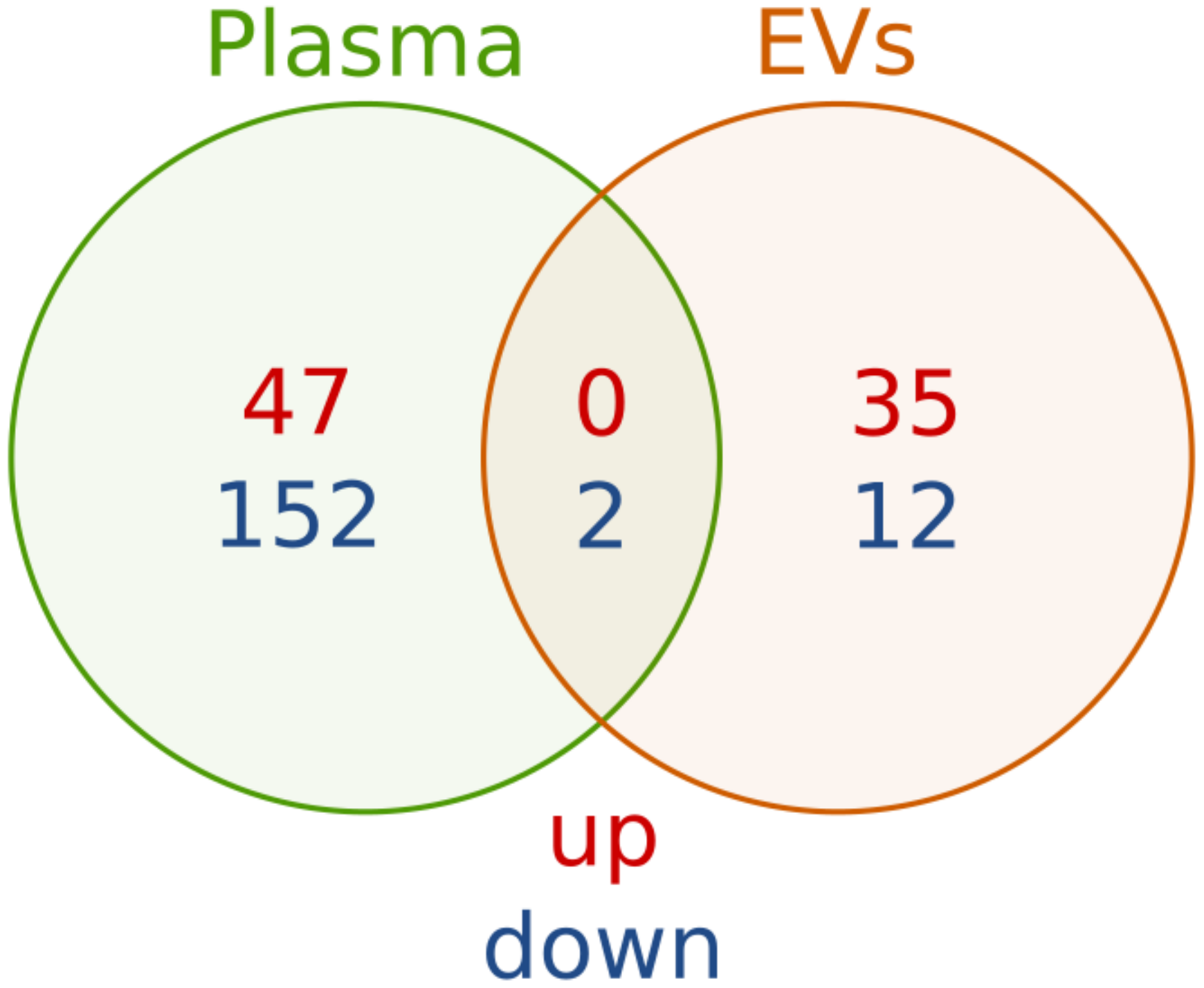

Figure 1

Venn diagram of differentially expressed miRNAs between ALL patients and control in plasma, EVs. The red color indicates up-regulated miRNAs, whereas the blue color indicates the down-regulated miRNAs. 

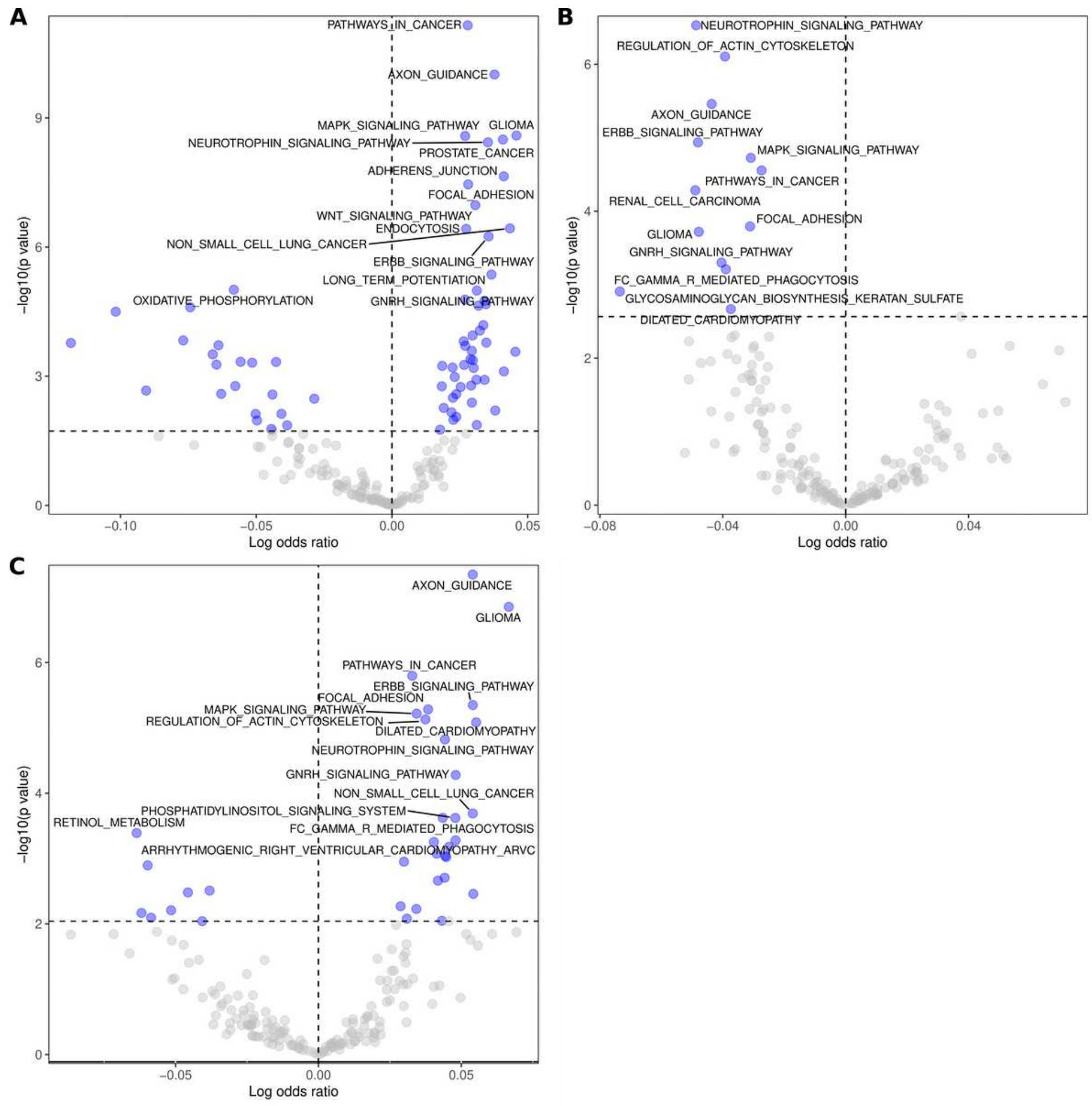

Figure 2

Results of the KEGG enrichment analysis among the targets of differentially expressed miRNAs in plasma (A), extracellular vesicles (B), and between the compartments (C). 


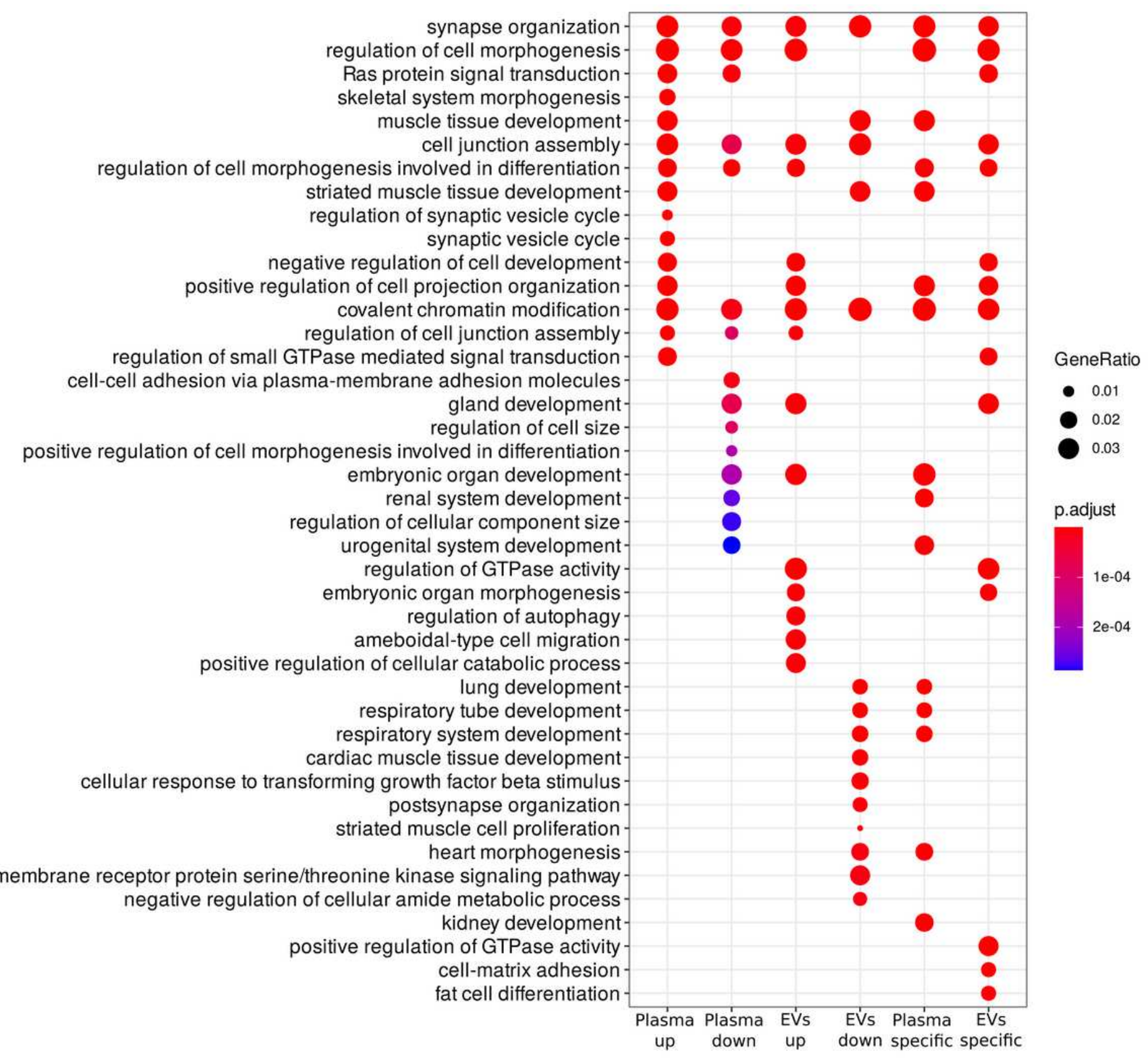

\section{Figure 3}

Results of the GO enrichment analysis (top 15 terms for each group) among targets of differentially expressed miRNAs in plasma, extracellular vesicles, and between the two compartments (plasma up analysis for miRNA up-regulated in plasma, plasma down - analysis for miRNA down-regulated in plasma, EVs up - analysis for miRNA up-regulated in EVs, EVs down - analysis for miRNA down-regulated in EVs, plasma specific - analysis for miRNA that abundance in plasma is higher than in EVs in comparison to controls, EVs specific - analysis for miRNA that abundance in EVs is higher than in plasma in comparison to controls ). 


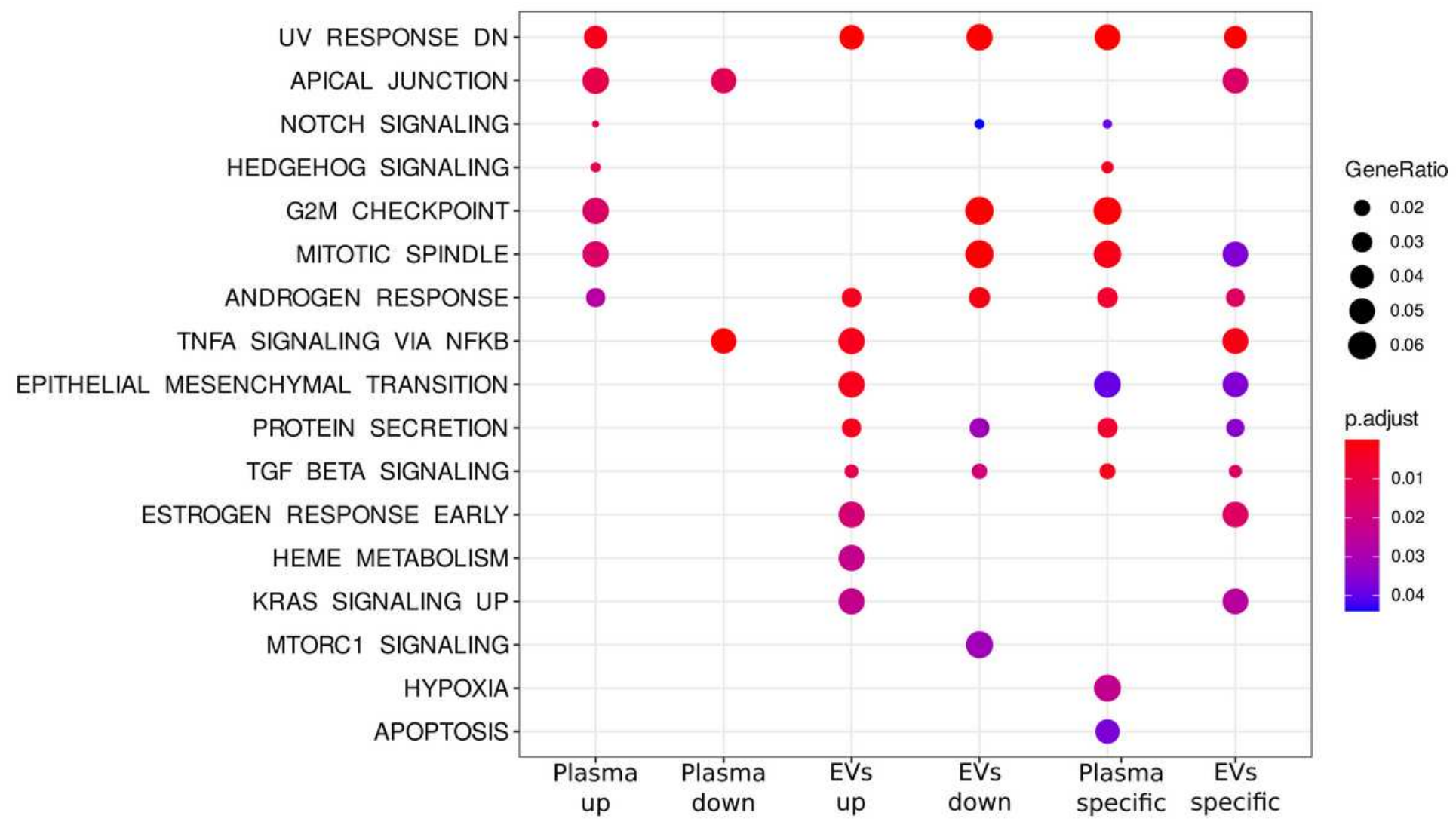

\section{Figure 4}

Results of the 'hallmark' enrichment analysis among targets of differentially expressed miRNAs in plasma, extracellular vesicles, and between the two compartments (plasma up - analysis for miRNA upregulated in plasma, plasma down - analysis for miRNA down-regulated in plasma, EVs up - analysis for miRNA up-regulated in EVs, EVs down - analysis for miRNA down-regulated in EVs, plasma specific analysis for miRNA that abundance in plasma is higher than in EVs in comparison to controls, EVs specific - analysis for miRNA that abundance in EVs is higher than in plasma in comparison to controls ) . 
A

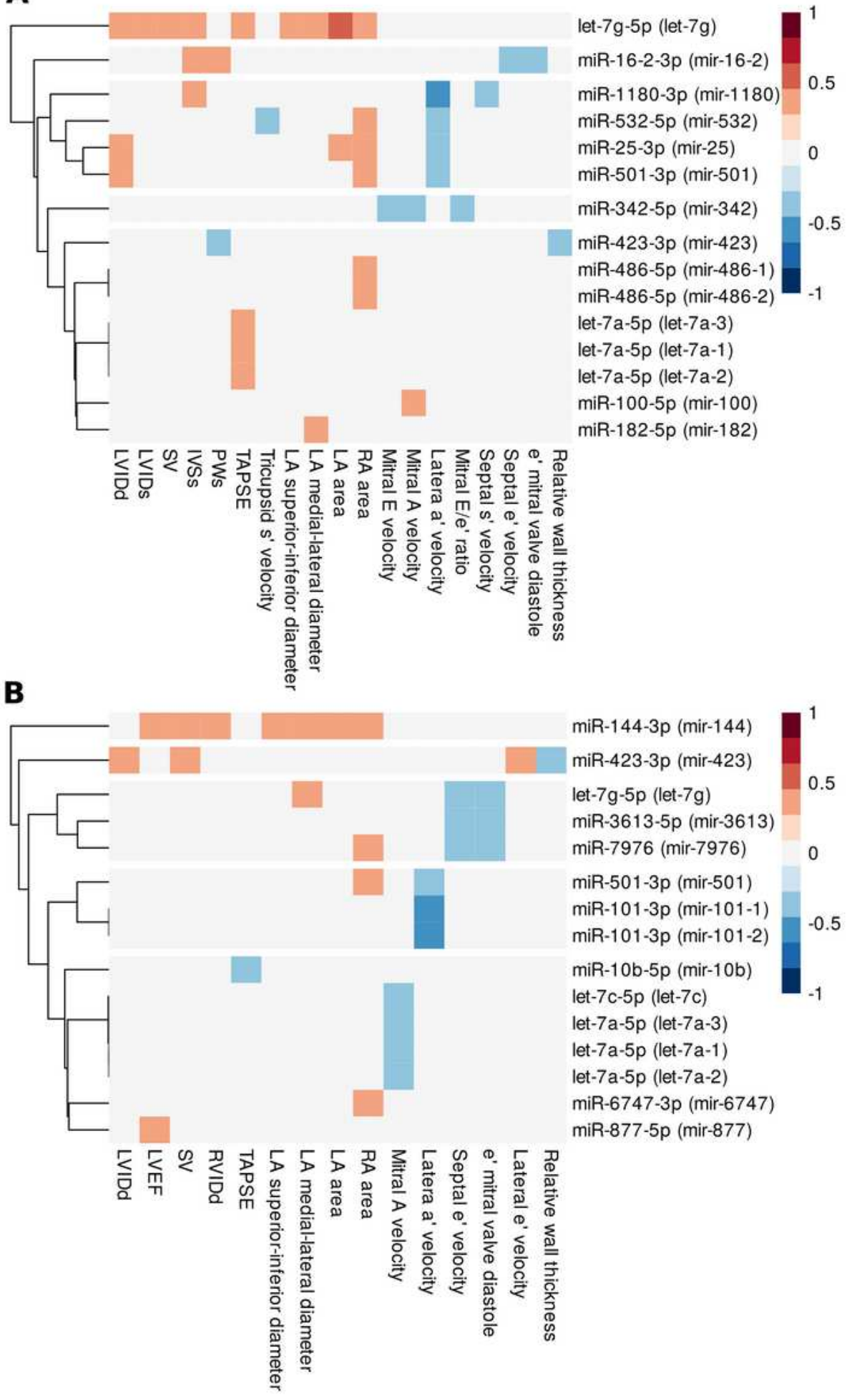

Figure 5

Correlation of the miRNAs, with dissimilar variance in plasma $(A)$ and EVs (B) between control and ALL patients, with echocardiography parameters. The miRNAs without significant correlation were removed. Non-significant correlation was presented as white blocks. (LVID - left ventricular internal dimension in systole (s) or diastole (d), LVEF - left ventricular ejection fraction, SV - left ventricular stroke volume, IVSs - septal wall thickness, PWs - left ventricular posterior wall thickness, RVID - right ventricular internal 
dimension, TAPSE - tricuspid annular plane systolic excursion, LA area-left atrial area, RA - right atrial area) 